FERMILAB-Conf-96/438-E

CDF

\title{
Beauty Baryons: Recent CDF Results
}

\author{
J. Tseng \\ For the CDF Collaboration \\ Fermi National Accelerator Laboratory \\ P.O. Box 500, Batavia, Illinois 60510 \\ Massachusetts Institute of Technology \\ Cambridge, Massachusetts 02139
}

December 1996

Published Proceedings of the 2 nd International Conference on Hyperons, Charm and Beauty Hadrons, Montreal, Quebec, August 27-30, 1996 


\section{Disclaimer}

This report was prepared as an account of work sponsored by an agency of the United States Government. Neither the United States Government nor any agency thereof, nor any of their employees, makes any warranty, expressed or implied, or assumes any legal liability or responsibility for the accuracy, completeness, or usefulness of any information, apparatus, product, or process disclosed, or represents that its use would not infringe privately owned rights. Reference herein to any specific commercial product, process, or service by trade name, trademark, manufacturer, or otherwise, does not necessarily constitute or imply its endorsement, recommendation, or favoring by the United States Government or any agency thereof. The views and opinions of authors expressed herein do not necessarily state or reflect those of the United States Government or any agency thereof.

\section{Distribution}

Approved for public release; further dissemination unlimited. 


\title{
Beauty Baryons: Recent CDF Results
}

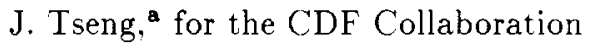 \\ ${ }^{a}$ Massachusetts Institute of Technology, \\ Cambridge, Massachusetts 02139, LSA
}

Using data collected between 1992 and 1995 at the Fermilab Tevatron, CDF has searched for the $\Lambda_{b}$ baryon through both semileptonic and hadronic decay channels. This presentation reviews measurements of the $\Lambda_{b}$ mass, lifetime, and production and decay rates performed with this data.

The Fermilab Tevatron $\bar{p} p$ collider, with centerof-mass energy $\sqrt{s}=1800 \mathrm{GeV}$, produces copious numbers of $b$ quarks - some eighty thousand million between 1992 and 1995 --and is thus a useful tool for the study of $b$-quark physics. It is natural, therefore, having found and studied mesons containing $b$ quarks, to search the data for the presence of baryons containing such quarks. This article describes recent studies concerning the lowest lying baryon, the $\Lambda_{b}$, conducted at the Collider Detector at Fermilab (CDF). [1]

\section{Full Reconstruction: $\Lambda_{b} \rightarrow J / \psi 1^{0}$}

One of the signatures proven most useful for $b$ hadron reconstruction at CDF is the decay $J / \psi \rightarrow \mu^{+} \mu^{-}$, because the two muons readily distinguish the event from the far more common light-quark processes that occur at a hadron collider. The muons are identified by matching tracks in the Central Tracking Chamber (CTC) with track stubs in wire chambers placed outside the electromagnetic and hadronic calorimeters. These outer chambers cover the pseudorapidity range $|\eta|<1$ relative to the nominal interaction point. The tracks are required to have momentum, $p_{T}$, measured in the detector plane transverse to the beam, of at least $2 \mathrm{GeV} / c$. The two muons are vertex constrained as well as mass constrained to the world average $J / \psi$ mass. [2] and the resulting probability is required to exceed $0.5 \%$.

The search for $\Lambda_{b}$ in the resulting data sample is for its decay to $J / \psi \Lambda^{\circ}$. [3] (In this article, charge conjugate decays are implied.) The de- cay $B^{\circ} \rightarrow J / \psi K_{s}^{0}$, followed by $K_{s}^{\circ} \rightarrow \pi^{+} \pi^{-}$, is topologically very similar to $\Lambda_{b} \rightarrow J / \psi \Lambda^{0}$ and is used to check the mass measurement as well as normalize the rate measurement.

The $\Lambda^{0}$ is reconstructed through its decay to $p \pi^{-}$. Because of the large mass difference between the proton and pion, the proton mass is assigned to the higher- $p_{T}$ track. The $\Lambda^{0}$ vertex is required to have a transverse displacement, projected along its momentum, of at least $1.0 \mathrm{~cm}$ beyond the primary vertex. The total $p_{T}$ of the $\Lambda^{0}$ must be at least $1.5 \mathrm{GeV} / c$. The two tracks are also required to have $d E / d x$ measurements in the CTC within $2 \sigma$ of their predicted energy loss. Combinations with mass within $4 \mathrm{MeV} / c^{2}$ (about $2 \sigma$ ) of the world average for $\Lambda^{0}$ are taken to be $\Lambda^{0}$ candidates. The event is removed from consideration if assigning the proton candidate a pion mass results in a combined mass within $12 \mathrm{MeV} / \mathrm{c}^{2}$ of the $K_{s}^{0}$ mass.

The $J / \psi$ and $\Lambda^{0}$ candidates are then combined, constraining the dimuon pair to the $J / \psi$ mass and the $\Lambda^{0}$ to point back to the dimuon vertex. The combined $p_{T}$ is required to exceed $6.0 \mathrm{GeV} / c^{2}$ and have $|\eta|<1$ relative to the primary interaction point. In addition, a lifetime cut, $c \tau>100 \mu \mathrm{m}$, is applied to reduce non- $b$ backgrounds. The resulting mass distribution is shown in Figure 1.

\subsection{Mass Measurement}

The above reconstruction method has been tested in parts on several established signals such as $\psi(2 S) \rightarrow J / \psi \pi^{+} \pi^{-}, \chi_{c 1,2} \rightarrow J / \psi \gamma$ with photon conversion $\gamma \rightarrow e^{+} e^{-}, \Xi^{-} \rightarrow \Lambda^{0} \pi^{-}$, and, as 


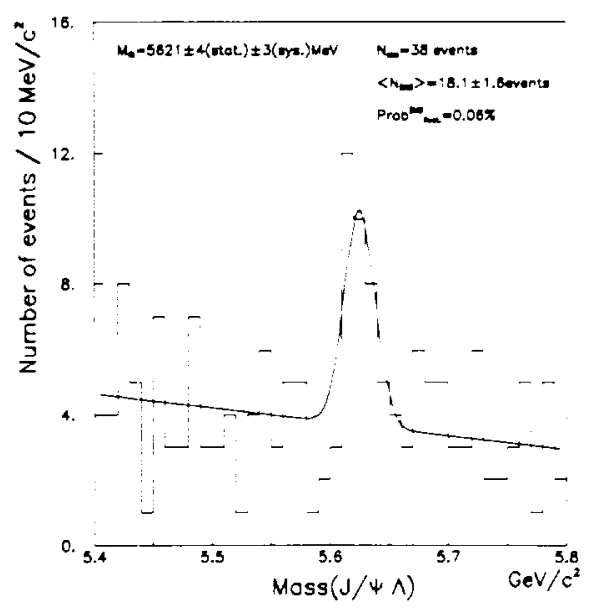

Figure 1. Invariant mass distribution of $J / \psi \Lambda^{\circ}$ candidates. The fit results are from an unbinned maximum likelihood fit to a gaussian signal over a linear background.

mentioned above, the topologically similar $B^{0} \rightarrow$ $J / \psi K_{s}^{0}$. No systematic mass effects were uncovered with these checks.

'The statistical significance of the peak is estimated by the probability that a linear background could fluctuate up to the observed number of events in five consecutive bins, which corresponds to the mass resolution of the $\Lambda_{b}$ determined by Monte Carlo. The search window was defined in several ways, and all methods yicld a significance of about $3 \sigma$.

The mass of this signal is $5621 \pm 4 \mathrm{MeV} / \mathrm{c}^{2}$ from an unbinned maximum likelihood fit. Systematic uncertanties due to detector effects and nonuniformities are measured to be $3 \mathrm{MeV} / \mathrm{c}^{2}$. The final mass result is

$$
m_{\Lambda_{b}}=5621 \pm 4(\text { stat }) \pm 3(\text { sys }) \mathrm{MeV} / \mathrm{c}^{2} .
$$

Figure 2 shows this value in comparison with recent measurements from CA1. DELPHI, and ALEPH. [4] 5] Calculating the mass difference between the $\Lambda_{b}$ and the $B^{0}$ almost eliminates the

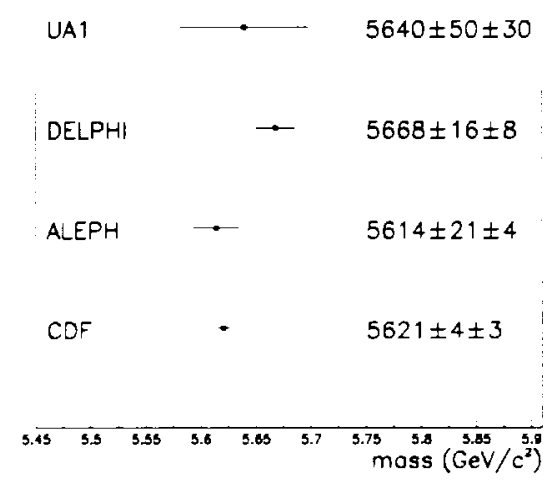

Figure 2. Recent $\Lambda_{b}$ mass measurements.

systematic uncertainties. That difference is

$$
m_{\Lambda_{\mathrm{b}}}-m_{B^{\circ}}=340 \pm 5(\text { stat }) \pm 1(\text { sys }) \mathrm{MeV} / c^{2} .
$$

\subsection{Production and Decay Rate}

As mentioned above, the $\Lambda_{b}$ production and decay rate can be measured relative to that of $B^{0} \rightarrow J / \psi K_{s}^{0}$. The reconstruction algorithms are essentially the same, and hence many systematic uncertainties largely cancel in the ratio.

Additional quality cuts are imposed upon the data in order to perform the measurement where efficiencies are well understood. These cuts include the requirement that all tracks have $p_{T}>$ $400 \mathrm{MeV} / \mathrm{c}$ and the muons must be reconstructed using precision measurements in the Silicon Vertex Detector (SVX). [6] The ratio of efficiencies of these cuts is measured in Monte Carlo to be

$$
\frac{\epsilon_{B^{\circ}} \operatorname{Br}\left(K_{s}^{0} \rightarrow \pi^{+} \pi^{-}\right)}{\epsilon_{\Lambda_{b}} \operatorname{Br}\left(\Lambda^{0} \rightarrow p \pi^{-}\right)}=2.02 \pm 0.05 .
$$

After these cuts the signals are as shown in Figure 3 . There are $7.7 \pm 3.4 \Lambda_{b}$ events and $57.6 \pm 8.7$ $B^{0}$ 's. The ratio of production and decay rates is therefore

$$
\frac{\sigma\left(\bar{p} p \rightarrow \Lambda_{b} X\right) \operatorname{Br}\left(\Lambda_{b} \rightarrow J / \psi \Lambda^{0}\right)}{\sigma\left(\bar{p} p \rightarrow B^{0} X\right) \operatorname{Br}\left(B^{0} \rightarrow J / \psi K_{\bullet}^{0}\right)}
$$




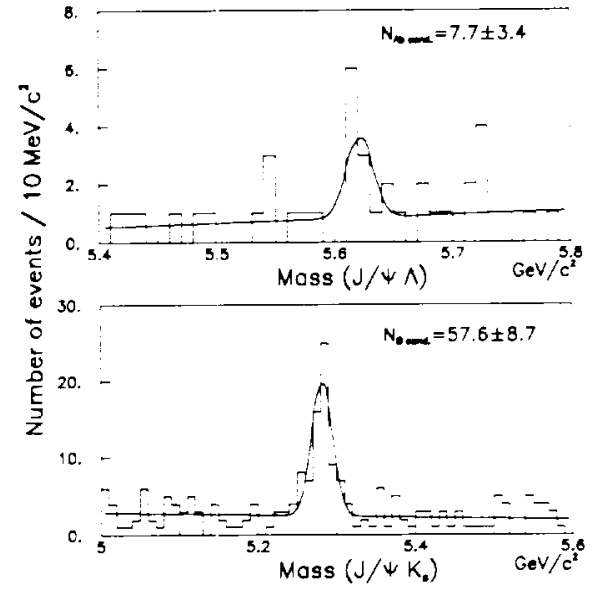

Figure 3. $\Lambda_{b} \rightarrow J / \psi \Lambda^{0}$ and $B^{0} \rightarrow J / \psi K_{s}^{0}$ mass distributions for the rate measurement.

$$
=0.27 \pm 0.12(\text { stat }) \pm 0.05 \text { (sys) } .
$$

The largest systematic uncertainties are due to the fragmentation and decay dynamics of the $\Lambda_{b}$.

\section{Semileptonic Decay: $\Lambda_{b} \rightarrow \Lambda_{c}^{+} \ell^{-} \bar{\nu}_{\ell} X$}

Partial reconstruction of the semileptonic decay of the $\Lambda_{b}$ is another approach to the study of the $b$ baryon, and it offers several advantages. among them a large branching fraction on the order of $10 \%$, and, because of the high thresholds on the single lepton triggers at CDF, favorable kinematics for distinguishing the high energy signal from the relatively low energy backgrounds.

The signature for $\Lambda_{b}$ semileptonic decay used in the present analyses is a "right sign" charmlepton pair $\left(\Lambda_{c}^{+} \ell^{-}\right.$and $\left.\Lambda_{c}^{-} \ell^{+}\right)$with invariant mass consistent with $\Lambda_{b}$ decay. The $\Lambda_{c}^{+}$is fully reconstructed through its decay to $p K^{-} \pi^{+}$. "Wrong sign" pairs $\left(\Lambda_{c}^{+} \ell^{+}\right.$and $\left.\Lambda_{c}^{-} \ell^{-}\right)$, which cannot come from semileptonic $\Lambda_{b}$ decay, are used for background studies.

The CDF trigger selects electrons with electromagnetic energy deposition in the central detector region of at least $9 \mathrm{GeV}$ and pointed to by a
CTC track. The electron candidates are further purified by examining their deposition profiles in the calorimeter as well as in strip chambers embedded within the calorimeter. Muon candidates are selected as described in Section 1 but with $p_{T}>9 \mathrm{GeV} / c$.

The $\Lambda_{c}^{+}$is reconstructed as a three-prong vertex with all tracks in the SVX. 'The $p_{T}$ cuts on the proton, kaon, and pion are set at 2,1 , and $0.45 \mathrm{GeV} / c$. Since both the $\Lambda_{b}$ and $\Lambda_{c}^{+}$are longlived particles, cuts on the apparent flight distance of the vertex and the inpact parameters of the tracks further suppress the background while keeping most of the signal. The proton $d E / d x$ is required to be within $2 \sigma$ of expectation. The $\Lambda_{c}$ candidate is then combined with the lepton, and the invariant mass of the two particles is calculated and required to be between 3.5 and $6 \mathrm{GeV} / c^{2}$. The lower limit cuts out part of the kinematically allowed region but suppresses backgrounds from $B$ meson decays.

\subsection{Production and Decay Rate}

The reconstruction program described above was first carried out in the single electron data of the first $19.3 \mathrm{pb}^{-1}$. The $p K \pi$ mass distribution is shown in Figure 4 for the "right sign" combinations, and Figure 5 for the "wrong sign." Monte Carlo studies show that with the above cuts, backgrounds from $\Lambda_{c}^{+}$duplicates (switching the proton and pion), reflections from other charm hadrons, $B$ meson and non-semileptonic $A_{b}$ decays, and pairs from different quarks do not contribute significantly to the size of the "right sign" signal. The product of cross section and branching fractions is measured with this signal to be

$$
\begin{gathered}
\sigma_{b}\left(p_{T}^{b}>10.5 \mathrm{GeV} / c,|y|<1\right) \times \\
f_{\Lambda_{b}} \operatorname{Br}\left(\Lambda_{b} \rightarrow \Lambda_{c}^{+} e^{-} \bar{\nu}_{e} X\right) \operatorname{Br}\left(\Lambda_{c} \rightarrow p K^{-} \pi^{+}\right) \\
=\left(1.9 \pm 0.51(\text { stat }) \pm 0.36(s y s)_{-0.56}^{+0.66}(t h)\right) \mathrm{nb}
\end{gathered}
$$

where $f_{\Lambda_{b}}$ is the probability that the fragmentation of $a b$ quark produces $a b$ baryon which then yields the "right sign" signature; here " $\Lambda_{b}$ " designates any such baryon, the $\Lambda_{b}$ itself being expected to contribute the largest part. The systematic uncertainties are mostly those due to detector simulation, whereas the theoretical uncer- 


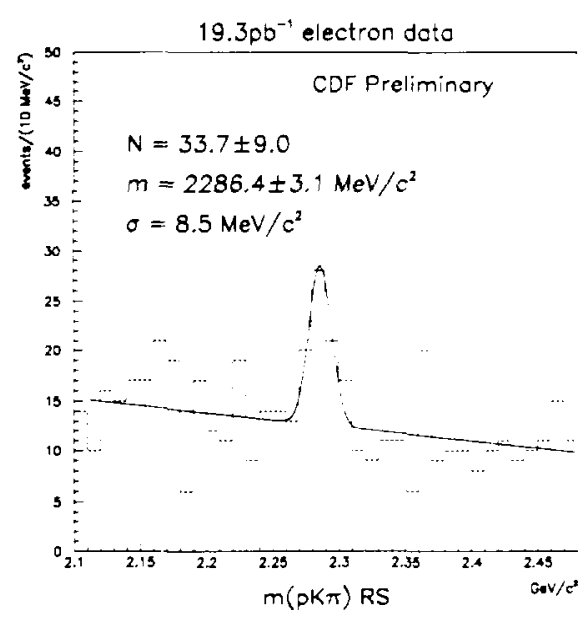

Figure 4. "Right sign" $p K \pi$ mass distribution.

tainties are mostly from uncertainties in fragmentation and the decay model used in the efficiency calculation.

The above rate measurement can be converted into a product of branching fractions by dividing it by the $b$ quark cross section measured at CDF for the specified kinematic region, [7]

$$
\begin{gathered}
\sigma_{b}\left(p_{T}^{b}>10.5 \mathrm{GeV} / c,|y|<1\right) \\
\quad=1.99 \pm 0.30 \pm 0.41 \mu \mathrm{b} .
\end{gathered}
$$

Removing common systematic uncertainties, the product of branching fractions is

$$
\begin{aligned}
& f_{\Lambda_{b}} \operatorname{Br}\left(\Lambda_{b} \rightarrow \Lambda_{c}^{+} e^{-} \bar{\nu}_{e} X\right) \operatorname{Br}\left(\Lambda_{c} \rightarrow p K^{-} \pi^{+}\right) \\
= & (9.5 \pm 2.5(\text { stat }) \pm 2.9(\text { sys }) \\
-2.8 .3 & (\text { th })) \times 10^{-4},
\end{aligned}
$$

which is higher than but consistent with previous results from LEP. [8]

\subsection{Lifetime Measurement}

The $\Lambda_{b}$ lifetime has been measured using semileptonic decays at LEP and is notable for being unexpectedly short. QCD predictions place the lifetime ratio $\tau_{\Lambda_{b}} / \tau_{B^{\circ}}$ at not much less than $0.9 ;[9]$ as shown in Figure 6 , the observed ratio is $0.73 \pm 0.06$, averaging over analyses based upon sign correlations in $\Lambda_{c} \ell, \Lambda \ell$, and p pairs. [10]

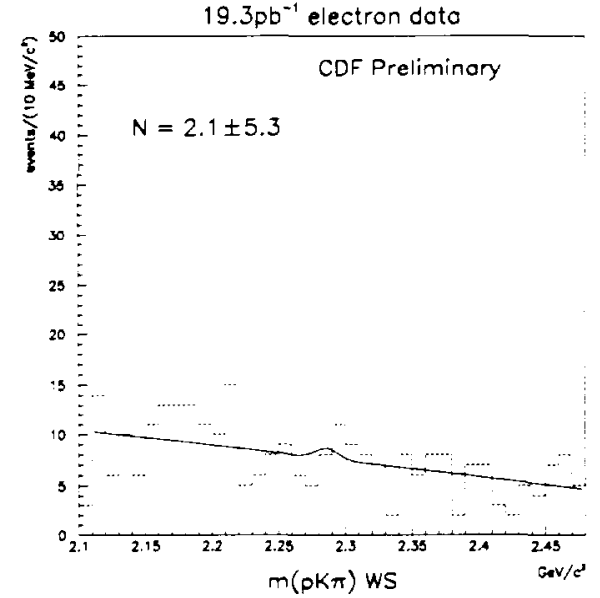

Figure 5. "Wrong sign" $p K \pi$ mass distribution.

Such a short lifetime is difficult to accomodate theoretically. [11]

The $\Lambda_{b}$ lifetime is measured at CDF with electrons and muons from $110 \mathrm{pb}^{-1}$ of data collected from 1992 to 1995 . [12] The analysis uses cuts similar to those used in the rate measurement. The "right sign" and "wrong sign" $p K \pi$ mass distributions are shown in Figure 7. There are $197 \pm 25$ "right sign" events, and again no discernible peak among the "wrong sign" combinations.

The lifetime measurement is performed by measuring the transverse distance from the primary interaction point to the $\Lambda_{c} \ell$ vertex. The distribution of "pseudo-proper decay lengths,"

$$
c \tau^{\prime}=L_{x y} \frac{M}{p_{T}\left(\Lambda_{c} \ell\right)},
$$

so called because the neutrino momentum is not included, is shown in Figure 8. The effect of the missing neutrino is modeled in Monte Carlo and is seen to be largely insensitive to $p_{T}\left(\Lambda_{c} \ell\right)$ and lepton $p_{T}$. The correction is convoluted with the lifetirne fit to give the true $c \tau$. The lifetime fit simultaneously fits the signal and background shapes and gives

$$
\tau_{\Lambda_{b}}=1.32 \pm 0.15(\text { stat }) \pm 0.07 \text { (sys) ps }
$$




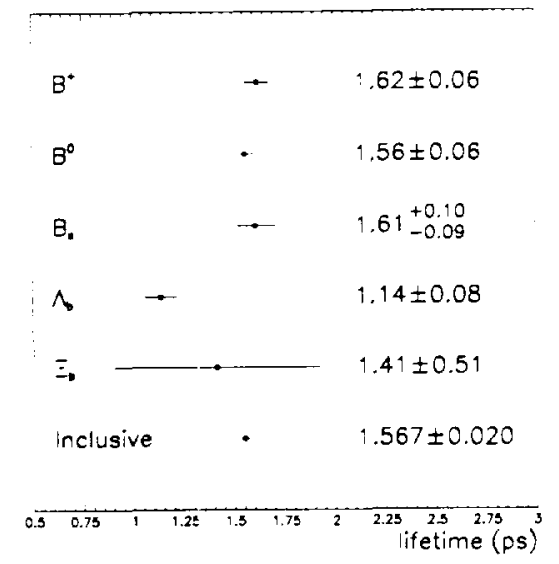

Figure 6 . Comparison of $b$ hadron lifetimes.

where the systematic uncertainties include variations in the background shape and fitting procedure, possible bias due to the event selection cuts, and uncertainties in $\Lambda_{b}$ production and decay, including the effect of additional daughter hadrons. The lifetime ratio is therefore

$$
\frac{\tau_{\Lambda_{b}}}{\tau_{B^{\circ}}}=0.8 .5 \pm 0.10(\text { stat }) \pm 0.05(\text { sys }) \text {. }
$$

The CDF lifetime measurement is compared in Figure 9 with other measurements which fully reconstruct the $\Lambda_{c}^{+}$and is seen to be consistent with them. [13] Considered alone, it is also in good agreement with the theoretical expectation.

\section{Conclusion}

In data collected at the Tevatron between 1992 and 1995, CDF has obscrved the $\Lambda_{b}$ through its hadronic decay to $J / \psi \Lambda^{0}$ and semileptonic decay to $\Lambda_{c}^{+} \ell^{-} \bar{\nu}_{\ell} X$. Its mass is measured to be $5621 \pm 4$ (stat) \pm 3 (sys) $\mathrm{MeV} / \mathrm{c}^{2}$ and its lifetime to be $1.32 \pm 0.15$ (stat) \pm 0.07 (sys) ps. The lifetime ratio, $\tau_{\Lambda_{b}} / \tau_{B^{\circ}}=0.85 \pm 0.10$ (stat) \pm 0.05 (sys), is in agreement with theoretical expectations as well as recent LEP measurements. In addition, the production and decay rates are measured to

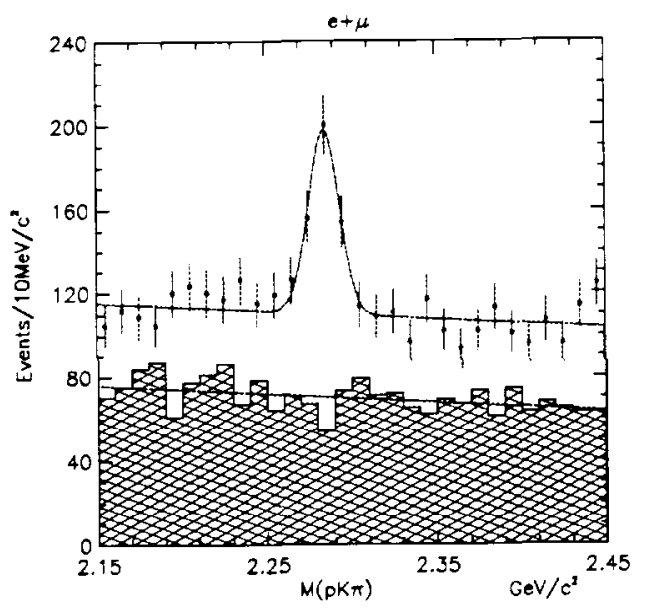

Figure 7. "Right-sign" (points) and "wrong-sign" (hatched histogram) $p K \pi$ mass distributions.

be

$$
\begin{aligned}
& \frac{\sigma\left(\bar{p} p \rightarrow \Lambda_{b} X\right) \operatorname{Br}\left(\Lambda_{b} \rightarrow J / \psi \Lambda^{0}\right)}{\sigma\left(\bar{p} p \rightarrow B^{0} X\right) \operatorname{Br}\left(B^{0} \rightarrow J / \psi K_{\iota}^{0}\right)} \\
& =0.27 \pm 0.12(\text { stat }) \pm 0.05(\text { sys })
\end{aligned}
$$

and, using the first $19.3 \mathrm{pb}^{-1}$ of electron data,

$$
\begin{gathered}
\sigma_{b}\left(p_{T}^{b}>10.5 \mathrm{GeV} / c,|y|<1\right) \times \\
f_{\Lambda_{b}} \operatorname{Br}\left(\Lambda_{b} \rightarrow \Lambda_{c}^{+} e^{-} \bar{\nu}_{e} X\right) \operatorname{Br}\left(\Lambda_{c} \rightarrow p K^{-} \pi^{+}\right) \\
=\left(1.9 \pm 0.51(\text { stat }) \pm 0.36(\text { sys })_{-0.56}^{+0.66}(\text { th })\right) \mathrm{nb}
\end{gathered}
$$

The next Tevatron collider run is scheduled to begin in 1999 with the benefit of the Main Injector upgrade. CDF is currently upgrading its detector to take advantage of the resulting higher luminosities, and one may expect a rich harvest of $b$ baryon results in the future.

\section{REFERENCES}

1. F. Abe, et al.(CDF Collaboration), Nucl. Instrum. Meth. A271, 387 (1988); F. Abe, et al.(CDF Collaboration), Phys. Rev. D50, 2966 (1994).

2. L. Montanet, et al., Phys. Rev. D50, 1173 (1994). 


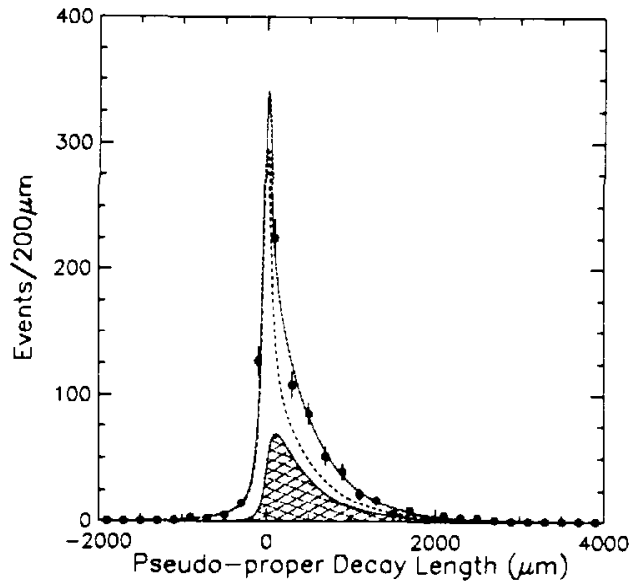

Figure 8. Distribution of pseudo-proper decay lengths $c \boldsymbol{\tau}^{\prime}$. The fit is from an unbinned maximum likelihood fit.

3. F. Abe, et al.(CDF Collaboration), FERMILAB-PLB-96-270-E, submit ted to Phys. Rev. D.

4. C. Albajar, et al.(LA1 Collaboration), Phys. Lett. B273, 540 (1991);

5. P. Abreu. et al.(DELLPHI Collaboration), Phys. Lett. B374, 351 (1996): D. Buskilic, et al.(ALEPH Collaboration), Phys. Lett. B380, 442 (1996).

6. D. Amidei, et al.., Nucl. Instrum. Meth. A350, 73 (1994); P. Azzi, et al.., Nucl. Instrum. Meth. A360, 137 (1995).

7. M.W. Bailey, Ph.D. thesis, Purdue Lniversity, 1994.

8. D. Buskilic, et al.(ALEPH Collaboration), Phys. Lett. B294, 145 (1992); $\Gamma$. Abreu, et al.(DELPHI Collaboration), $Z$. Phys. C68, 375 (1995); OPAL note OPN-082.

9. I.I. Bigi, et al., "Non-leptonic Decays of Beauty Hadrons," in S. Stone, ed., B Decays. World Scientific Publishing, p. 132, 1994.

10. R.M. Barnett, et al., Phys. Rev. D54, 1 (1996); P. Abreu, et al.(DELPHI Collaboration), Z. Phys. C68, 541 (1995): D. Buskilic,

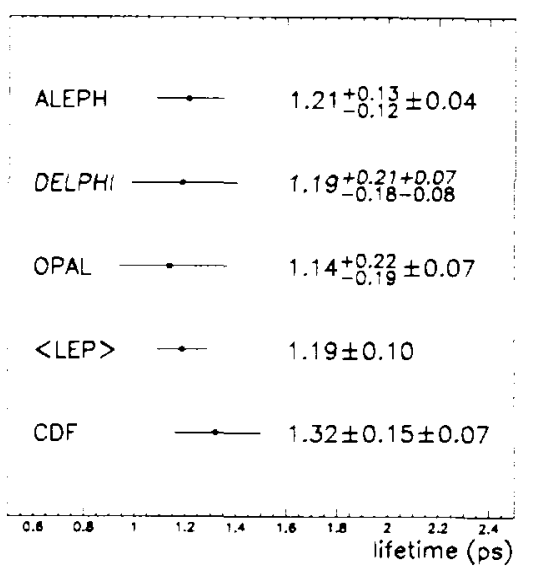

Figure 9. Comparison of $\Lambda_{b}$ lifetime measurements with fully reconstructed $\Lambda_{c}^{+}$'s.

et al.(ALEPH Collaboration), Phys. Lett. B384, 449 (1996).

11. M. Neubert, C.T. Sachrajda, CERN-TH-9619, submitted to Nucl. Phys. B; J. Rosner. Phys. Lett. B379, 267 (1996).

12. F. Abe, et al. (CDF Collaboration), Phys. Rev. Lett. 77, 1439 (1996).

13. The ALEPHI Collaboration, ICHEP96. PA01068, contributed to the 28th Intl. Conf. on High Energy Physics, Warsaw, Poland, 25-31 July 1996; P. Abreu, et al.(DELPHI Collaboration), Z. Phys. C71, 199 (1996); R. Akers, et al.(OPAL Collaboration), Z. Phys. C69, 195 (1996). 